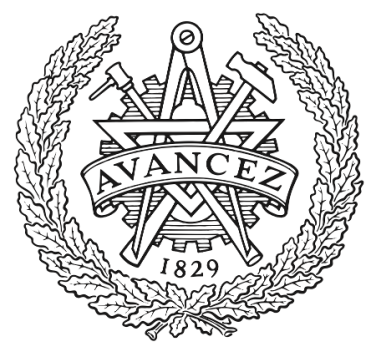

CHALMERS

UNIVERSITY OF TECHNOLOGY

\title{
Dielectric disorder in two-dimensional materials
}

Downloaded from: https://research.chalmers.se, 2023-04-26 13:53 UTC

Citation for the original published paper (version of record):

Raja, A., Waldecker, L., Zipfel, J. et al (2019). Dielectric disorder in two-dimensional materials.

Nature Nanotechnology, 14(9): 832-837. http://dx.doi.org/10.1038/s41565-019-0520-0

N.B. When citing this work, cite the original published paper. 


\title{
Dielectric disorder in two-dimensional materials
}

\author{
Archana Raja $\oplus^{1,10,11 \star}$, Lutz Waldecker, 2,311, Jonas Zipfel ${ }^{4}$, Yeongsu Cho ${ }^{5}$, Samuel Brem $\odot^{6}$, \\ Jonas D. Ziegler ${ }^{4}$, Marvin Kulig ${ }^{4}$, Takashi Taniguchi ${ }^{7}$, Kenji Watanabe ${ }^{7}{ }^{7}$, Ermin Malic ${ }^{6}{ }^{6}$, \\ Tony F. Heinz ${ }^{2,3}$, Timothy C. Berkelbach ${ }^{8,9}$ and Alexey Chernikov ${ }^{4 \star}$
}

\begin{abstract}
Understanding and controlling disorder is key to nanotechnology and materials science. Traditionally, disorder is attributed to local fluctuations of inherent material properties such as chemical and structural composition, doping or strain. Here, we present a fundamentally new source of disorder in nanoscale systems that is based entirely on the local changes of the Coulomb interaction due to fluctuations of the external dielectric environment. Using two-dimensional semiconductors as prototypes, we experimentally monitor dielectric disorder by probing the statistics and correlations of the exciton resonances, and theoretically analyse the influence of external screening and phonon scattering. Even moderate fluctuations of the dielectric environment are shown to induce large variations of the bandgap and exciton binding energies up to the $100 \mathrm{meV}$ range, often making it a dominant source of inhomogeneities. As a consequence, dielectric disorder has strong implications for both the optical and transport properties of nanoscale materials and their heterostructures.
\end{abstract}

R econciling deviations of real materials from ideal models constitutes a central challenge in solid-state science and nanotechnology. For many decades, this has motivated intense efforts to either mitigate and avoid or control and exploit such imperfections. Semiconductor technology, in particular, has relied heavily on this paradigm ${ }^{1}$ through the control of chemical doping $^{2}$, alloy and structural disorder ${ }^{3}$, radiative and non-radiative trap centres ${ }^{4}$, polymorphism and polydispersity ${ }^{5}$, as well as strain inhomogeneities and local strain relaxation ${ }^{6}$. In all of these cases, a fundamental understanding of the origin and impact of the materials' imperfections has been key to unlocking their technological potential.

Importantly, as the dimensions of materials approach nanometre length scales, enhanced Coulomb interactions between charge carriers $^{7}$ due to quantum confinement ${ }^{1}$ and reduced dielectric screening ${ }^{8,9}$ begin to dominate the optical and electronic response. For semiconductors, this leads to the formation of tightly bound electron-hole pairs, or excitons, and to a significant renormalization of the free-particle bandgap ${ }^{1,7}$. In the context of imperfections, these consequences of the electronic interactions imply a fundamentally new form of disorder: dielectric disorder. In contrast to the wellknown chemical or structural inhomogeneities associated with perturbations within the material, dielectric disorder originates entirely from local fluctuations of the environmental permittivity, inducing a spatial modulation of the Coulomb interaction. Here, we introduce and illustrate the concept of dielectric disorder using monolayers of semiconducting transition-metal dichalcogenides (TMDCs) and demonstrate its strong impact on the electronic transitions and exciton propagation. We also provide a framework to understand an important mechanism for improved optoelectronic properties via the widespread technique of encapsulation in van der Waals heterostructures.

\section{Concept of dielectric disorder}

An overview of the general concept of dielectric disorder is presented in Fig. 1. As demonstrated in recent reports, a local change in the external dielectric constant $\varepsilon_{\text {ext }}$ modifies the exciton states ${ }^{10-13}$ and the free-particle bandgap ${ }^{11-14}$, which allows for non-invasive engineering of lateral heterojunctions within the same material ${ }^{12,15}$. With respect to the magnitude of the effective screening, however, the external surroundings of real materials are usually far from homogeneous. Under most circumstances, it is not reasonable to assume that the substrate is atomically flat and chemically pure, or that the surface of the material and the interfaces it forms are absolutely clean ${ }^{16}$. Instead, the substrate typically exhibits spatial fluctuations of the surface morphology and contains polarizable impurities, and the material's surroundings are at least partially covered by adsorbates ${ }^{17}$, as schematically illustrated in Fig. 1a. Although these individual imperfections can be associated with a number of specific phenomena, they all share one central implication-they produce local variations of the external dielectric permittivity, indicated by $\Delta \varepsilon_{\text {ext }}$. Both electron-electron and electron-hole interactions are affected, leading to spatial inhomogeneities of the freeparticle bandgap and exciton binding energies, respectively.

The main influence of the Coulomb interaction on electronic excitations is summarized in Fig. $1 \mathrm{~b}$ for monolayer $\mathrm{WS}_{2}$, a typical two-dimensional (2D) semiconductor. The calculations take into account the effect of dielectric screening-induced bandgap renormalization and numerically evaluate exciton binding energies using a modified thin-film potential in the effective mass approximation $^{8,9,13,18}$ (see Supplementary Section 7 for details). For excitons, the transition energies are presented for the ground and first excited states, denoted by their principal quantum numbers of $n=1$ and 2 , respectively. These are plotted as a function of the squared inverse of the external dielectric screening constant, in analogy to the analytical

${ }^{1}$ Kavli Energy NanoScience Institute, University of California Berkeley, Berkeley, CA, USA. 2Department of Applied Physics, Stanford University, Stanford, CA, USA. ${ }^{3}$ SLAC National Accelerator Laboratory, Menlo Park, CA, USA. ${ }^{4}$ Department of Physics, University of Regensburg, Regensburg, Germany. ${ }^{5}$ Department of Chemistry and James Franck Institute, University of Chicago, Chicago, IL, USA. ${ }^{6}$ Department of Physics, Chalmers University of Technology, Gothenburg, Sweden. ${ }^{7}$ National Institute for Materials Science, Tsukuba, Ibaraki, Japan. ${ }^{8}$ Department of Chemistry, Columbia University, New York, NY, USA. ${ }^{9}$ Center for Computational Quantum Physics, Flatiron Institute, New York, NY, USA. ${ }^{10}$ Present address: The Molecular Foundry, Lawrence Berkeley National Laboratory, Berkeley, CA, USA. "These authors contributed equally: Archana Raja, Lutz Waldecker. *e-mail: araja@lbl.gov; alexey.chernikov@regensburg.de 
a

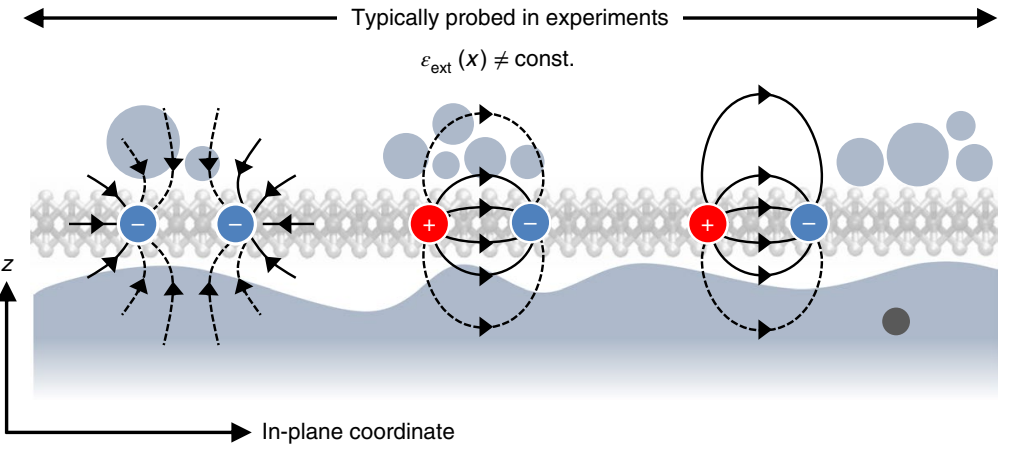

b

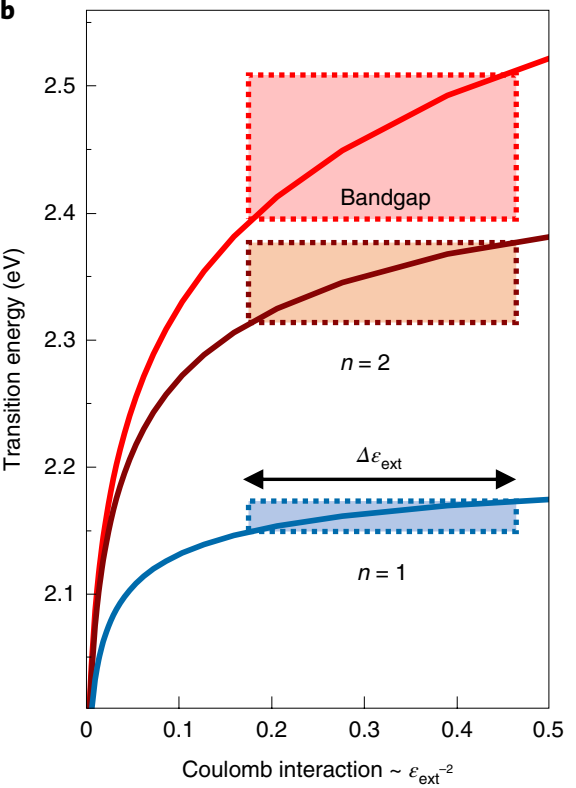

c

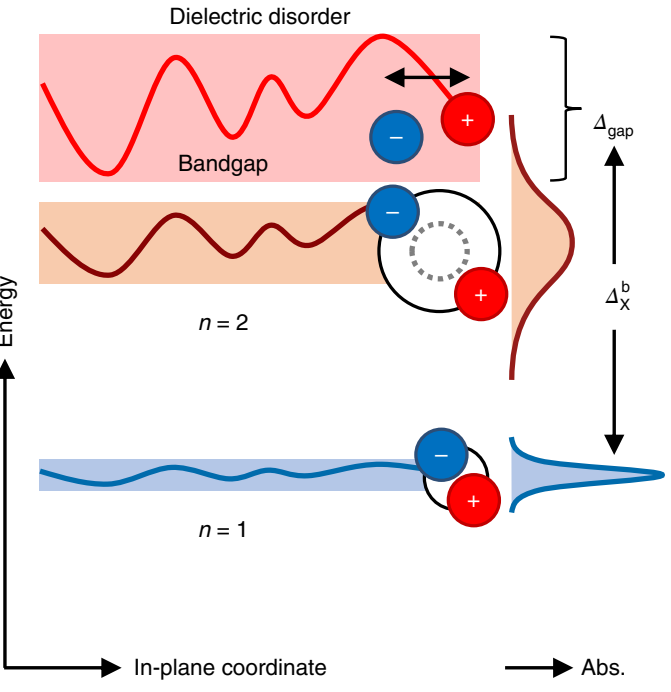

Fig. 1 | Dielectric disorder from spatial fluctuations of the dielectric screening. a, Schematic illustration of the electron-electron and electron-hole interaction being affected by environmental screening fluctuations due to the substrate roughness, impurities and adsorbates. b, Theoretically calculated energies of the bandgap and exciton states in a $\mathrm{WS}_{2}$ monolayer as a function of inverse squared external dielectric constant. Shaded areas indicate fluctuations from variations of the external screening. c, Illustration of the bandgap and exciton energy fluctuations due to dielectric disorder and the expected resonance broadening.

form of the exciton Rydberg energy ${ }^{8,9}$. With decreasing screening, the binding energies of the excitons increase and the bandgap shifts to higher energies.

Spatial fluctuations of the external screening should therefore directly induce local fluctuations of the bandgap and exciton energies. The consequences are illustrated schematically in Fig. 1c. The associated variations of the bandgap $\Delta_{\text {gap }}$ and exciton binding energy $\Delta_{\mathrm{X}}^{\mathrm{b}}$ can be very large, resembling the typical scale of the Coulomb effects on the order of hundreds of $\mathrm{meV}$ in mono- and few-layer TMDCs $^{19}$. These variations in the excitation energies should then appear either as spatially fluctuating peak positions or as characteristic inhomogeneous broadening of the relevant spectroscopic transitions if an effective ensemble area is probed. For the absolute energy of the exciton ground state, the two effects of the bandgap renormalization and exciton binding partially cancel each other out $^{19}$, resulting in a relatively small to moderate inhomogeneous broadening of the resonance. In comparison, the excited-state resonances, such as $n=2$, follow the external screening-induced variations of the bandgap to a much larger extent. Their absolute energies are strongly affected by the fluctuations of the dielectric screening and should thus be subject to increased characteristic inhomogeneous broadening.

\section{Exciton spectroscopy and correlation analysis}

To experimentally monitor dielectric disorder, we first analyse the linewidth statistics of both ground and excited exciton states through linear optical reflectance spectroscopy on a large number of $\mathrm{WS}_{2}$ and $\mathrm{WSe}_{2}$ monolayer samples. The exciton linewidths contain information on the inhomogeneous broadening within a probed area of about a square micrometre, and the distinctive correlations between the ground- and excited-state linewidths are used to clarify the underlying mechanism of dielectric disorder. The samples are exfoliated from commercially available bulk crystals and deposited on a variety of substrates. The specific details of the experimental techniques, sample preparation and analysis are discussed in Supplementary Sections 1-3, with a brief description in the Methods.

A representative low-temperature reflectance contrast spectrum of monolayer $\mathrm{WS}_{2}$ on a fused-silica substrate is presented in Fig. 2a. For the case of a monolayer on a transparent substrate, the reflectance contrast is roughly proportional to the optical absorption $^{20}$. The spectrum is dominated by the exciton $n=1$ resonance at $\sim 2.1 \mathrm{eV}$, typical for the intrinsic response of $\mathrm{WS}_{2}$ monolayers with negligible unintentional doping ${ }^{19}$. The signature of the first excited state $(n=2)$ is detected $\sim 160 \mathrm{meV}$ higher in energy. In stark 

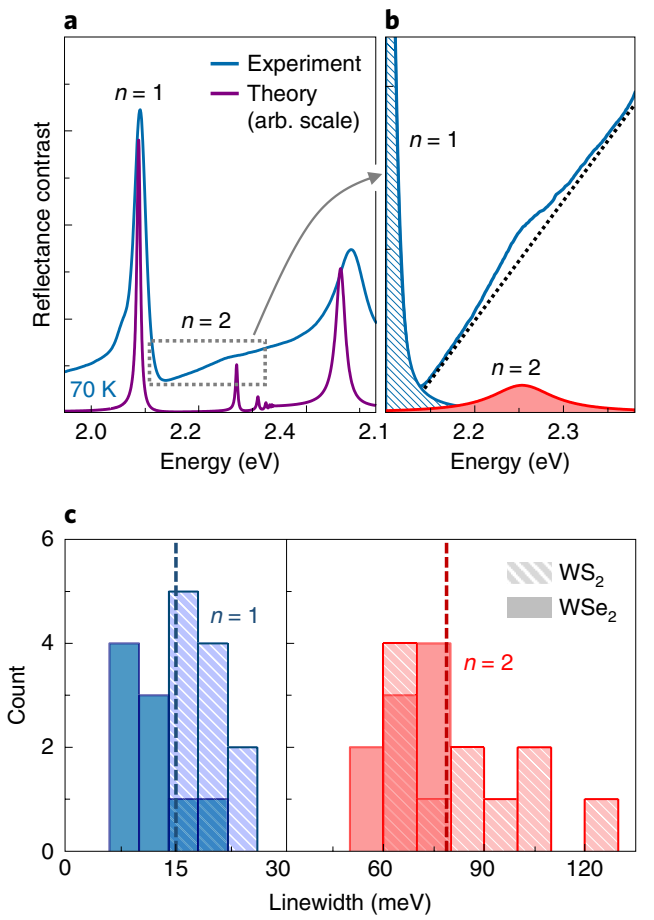

Fig. 2 | Low-temperature exciton linewidth statistics. a, Representative reflectance contrast spectrum of a $\mathrm{WS}_{2}$ monolayer on a fused-silica substrate at $T=77 \mathrm{~K}$. The result of a microscopic theory calculation, including purely homogeneous broadening from radiative and phononassisted scattering, is plotted below the experimental curve for comparison. b. Experimental data in the spectral range of the first excited state. c, Linewidth statistics of the exciton ground (left) and first excited state (right) across 22 monolayer flakes on glass substrates at cryogenic temperatures in the 5-77 K range. Dashed lines indicate corresponding mean values.

contrast to the prominent ground state, the amplitude of the signal from the excited-statepeakis extremelysmall, asfurtherhighlightedin Fig. 2b. Because the light-matter coupling of the $n=2$ transition is only about an order of magnitude lower than that of the $n=1$ peak $^{21,22}$ and is largely unaffected by temperature ${ }^{23}$, the observed dramatic difference in the signal heights is determined by the additional peak broadening of the excited-state resonance.

Interestingly, the homogeneous linewidths should be roughly the same for the ground and excited states due to the comparable rates of exciton-phonon scattering ${ }^{24}$, illustrated by the theoretically calculated spectrum obtained from microscopic calculations using the semiconductor Bloch equations approach ${ }^{25}$ and presented in Fig. 2a. In particular, relaxation towards the ground state provides only a minor contribution to the total scattering rate, similar to the behaviour associated with linewidth narrowing of excitons in bulk $\mathrm{Cu}_{2} \mathrm{O}$ with increasing principal quantum number $n$ (ref. ${ }^{26}$ ). The conclusion also agrees well with experiments on encapsulated samples $^{24}$, where low-temperature linewidths of the $n=2$ resonance can be as narrow as $3-5 \mathrm{meV}$, providing the limit for the intra-exciton relaxation rates (see Supplementary Section 6 for additional details on the experiment and theory).

The observation of spectrally broad excited states in as-exfoliated samples is further supported by low-temperature statistics obtained from 22 individual $\mathrm{WS}_{2}$ and $\mathrm{WSe}_{2}$ flakes on fused-silica and $\mathrm{SiO}_{2} / \mathrm{Si}$ substrates. The $n=1$ and 2 full-width at half-maximum linewidths are summarized as a histogram in Fig. 2c. The data are compiled from monolayers derived from different bulk crystals and prepared in different ways, including direct exfoliation and polymer stamping.
Nevertheless, the values for the exciton linewidths fall into two distinct categories: the ground-state resonance is relatively narrow, in the order of $15 \pm 5 \mathrm{meV}$, and the excited state is much broader with an average linewidth as large as $78 \pm 18 \mathrm{meV}$ in the studied sample set. Here, we note that contributions from higher excited states should not strongly affect the $n=2$ broadening, considering the energy separation from the $n=3$ state in the order of $60-70 \mathrm{meV}$ for non-encapsulated samples ${ }^{27,28}$. Altogether, such large inhomogeneous linewidths of the excited states directly correspond to at least comparable fluctuations of the exciton binding energy and the bandgap position.

Taking advantage of the characteristic relationship between the exciton peak energies with respect to screening, we are able to attribute the source of the broadening specifically to fluctuations of the dielectric environment. According to the theoretical predictions illustrated in Fig. 1 b, the shifts of the $n=1$ and 2 peak energies should be strongly correlated. The ratio of the relative shifts is presented in Fig. 3a. Correlations on a similar scale should therefore also appear between the resonance linewidths for an ensemble inhomogeneously broadened through dielectric disorder. Moreover, the ratio is also expected to depend on the average effective screening within the area of detection.

To test this prediction experimentally, we fabricated and studied a series of $\mathrm{WS}_{2}$ monolayers obtained from the same bulk crystal under identical measurement conditions and analysis procedures (see Methods and Supplementary Sections 1 and 3 for further details). The samples were either directly exfoliated onto polydimethylsiloxane (PDMS) films or subsequently stamped onto hexagonal boron nitride (hBN) substrates. Optical reflectance measurements were then performed at room temperature and under ambient conditions to avoid variations of pressure and unintentional condensates during cooling in a cryostat. For a quantitative analysis, we fitted and deconvoluted the exciton resonances to separate the inhomogeneous linewidth from the homogeneous broadening of $\sim 20 \mathrm{meV}$ in $\mathrm{WS}_{2}$ due to the exciton-phonon scattering, as previously discussed ${ }^{25}$ and further outlined in Supplementary Section 6.

The results, presented in Fig. $3 b$, demonstrate a strong correlation of the $n=2$ and 1 exciton peak broadening for both sets of samples. The ratios obtained from the linear fits are $3.7 \pm 0.1$ for the monolayers on PDMS and $2.2 \pm 0.1$ for monolayers on $\mathrm{hBN}$, in reasonable agreement with the theoretical expectations shown in Fig. 3a. In particular, the higher average external screening of the hBN substrates indeed leads to a smaller slope of the correlated inhomogeneous broadening. We further note that the correlations are already present both in the total room-temperature linewidths as well as in the low-temperature data (see Supplementary Section 5 for details).

These observations further allow us to exclude potential interpretations of the excited-state broadening as originating from alternative sources of disorder, such as doping or strain fluctuations. Strain predominantly leads to changes of the underlying freeparticle bandstructure ${ }^{29-33}$. For the studied samples, it would be necessary to assume already sizable strain fluctuations on the order of $0.25 \%$ even to account for the $n=1$ broadening. Most importantly, strain leads to shifts of the exciton ground and excited states that are equal in sign and magnitude, within several $\mathrm{meV}^{34}$, in stark contrast to our findings of much broader excited states compared to the ground state. Among more subtle effects, strain has been recently shown to influence homogeneous linewidths ${ }^{35,36}$. Considering that, the maximum estimated strain fluctuations of $0.25 \%$ would lead only to small additional linewidth changes on the order of $2-3 \mathrm{meV}$ (see Supplementary Sections 8 and 9 for more details on experimental scenarios where dielectric disorder is either dominant or suppressed).

We arrive at similar conclusions for the case of doping density fluctuations. Based on the observation of only a weak signature 

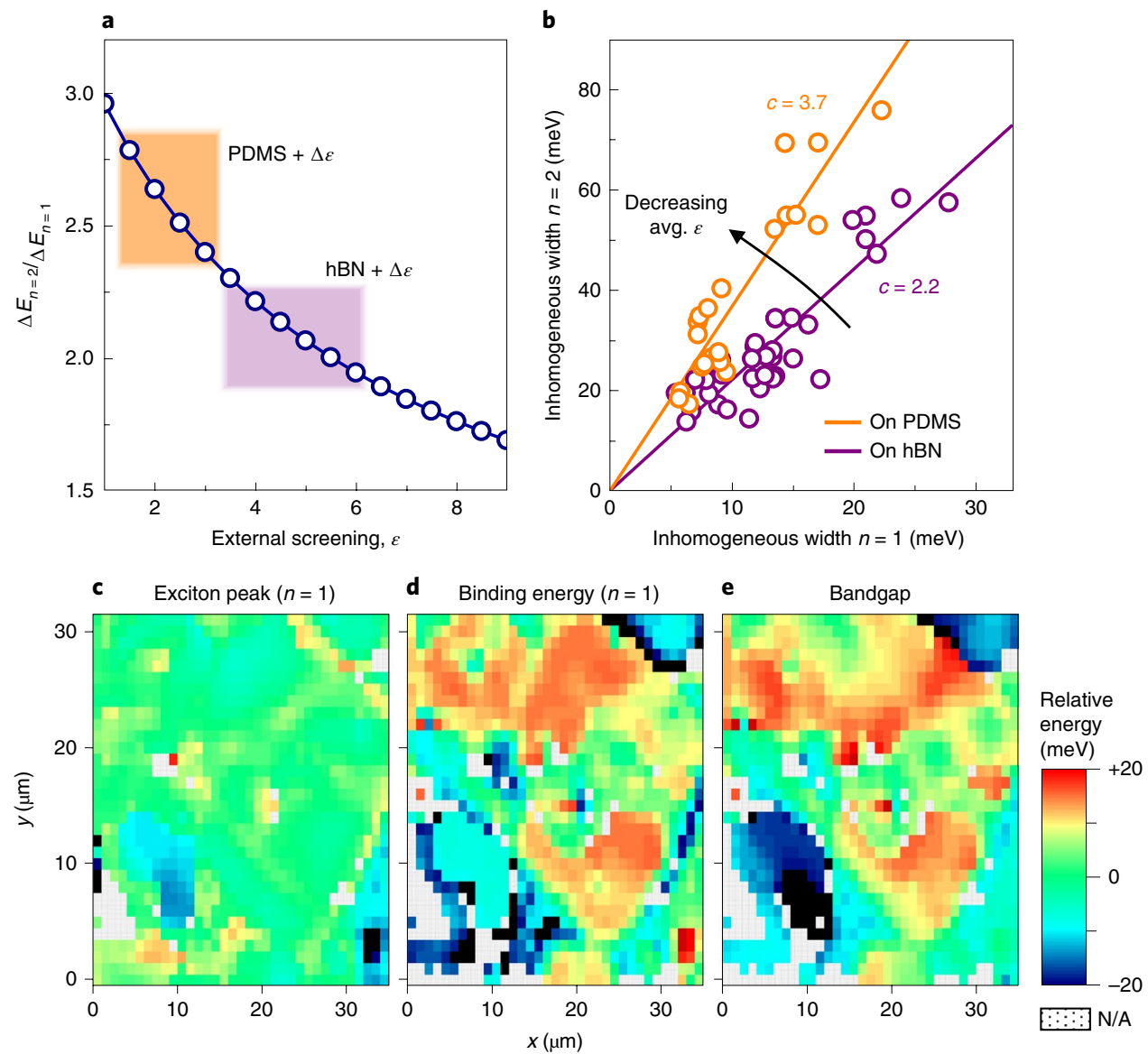

Fig. 3 | Correlation analysis and spatially resolved exciton spectroscopy. a, Ratio of the relative energy shifts of the $n=2$ and 1 transitions from calculations of the exciton binding energies and bandgap as a function of external dielectric screening. The typical screening range of $\mathrm{SiO}_{2}$ and hBN bulk substrates is

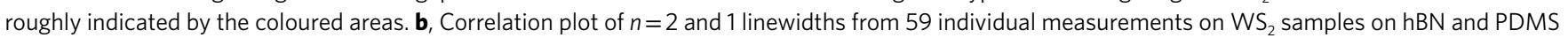
substrates. The linear fits are plotted along with corresponding slope parameters $c$. c. Fluctuations of the exciton $n=1$ peak position on optical length scales from hyperspatial reflectance spectroscopy of a hBN-encapsulated $\mathrm{WS}_{2}$ sample exhibiting spatial variations. $\mathbf{d}$, Corresponding fluctuations of the $n=1$ exciton binding energy obtained from the $n=2$ and 1 peak separation. e, Correlated fluctuations of the free-particle bandgap. Sample positions that do not allow for an unambiguous identification of the exciton peak energies are labeled as non-applicable (N/A) in figures $\mathbf{c}, \mathbf{d}$ and $\mathbf{e}$.

from the charged exciton state in our samples, the average doping fluctuations can be estimated to be on the order of $1 \times 10^{12} \mathrm{~cm}^{-2}$ or less $^{37-39}$. In that range, the changes of the $n=1$ and 2 optical transitions would be only a few $\mathrm{meV}$ in as-exfoliated $\mathrm{WS}_{2}$ samples ${ }^{39}$. Therefore, we also emphasize that the presented effects are not conceptually related to dipolar disorder ${ }^{40}$ describing the interactions of single carriers with randomly distributed permanent dipoles.

\section{Van der Waals heterostructures and exciton transport}

Importantly, dielectric disorder is shown to play a key role in the context of artificial van der Waals heterostructures. To illustrate dielectric fluctuations more directly, on optically accessible scales, we first chose a specific heterostructure where a $\mathrm{WS}_{2}$ monolayer was encapsulated between layers of hBN, yet the particular transfer resulted in pronounced fluctuations on an optically accessible micrometre scale, presumably due to varying interlayer contact. Using spatial reflectance mapping, we extracted the exciton binding and bandgap energies across the sample from the spectral positions of the exciton ground- and excited-state resonances (presented in Fig. $3 \mathrm{c}-\mathrm{e}$ ). Notably, the absolute energy of the exciton $n=1$ transition shows only small to moderate fluctuations. Much larger changes, however, are observed in the $n=1$ and 2 peak separations, corresponding to pronounced variations of the effective Coulomb interaction strength that is characteristic for dielectric disorder from local changes of the external dielectric screening. As a consequence, both free-particle bandgap and exciton binding energies exhibit prominent and correlated fluctuations across the sample (see Supplementary Section 8 for additional details).

On the other hand, a successful encapsulation with good interlayer contact typically results in accumulation of the adsorbates between the $\mathrm{hBN}$ and TMDC layers into hydrophobic pockets, leaving behind smooth, homogeneous areas that usually dominate the optical response ${ }^{41}$. It has thus been applied as a key strategy to prepare high-quality TMDC samples, following the original work on graphene $e^{42}$. The resulting linewidth narrowing ${ }^{43,44}$ is often generally ascribed to shielding of the material from the substrate and surface defects. Crucially, we argue that hBN-encapsulation with good interlayer contact primarily provides a homogeneous dielectric environment. As a consequence, it mitigates the effect of the dielectric disorder otherwise present in as-exfoliated samples and heterostructures with varying interlayer distance or trapped impurities.

The impact of this mechanism is directly demonstrated by comparing the optical spectra from a bare $\mathrm{WS}_{2}$ monolayer on a $\mathrm{SiO}_{2}$ substrate and a flake encapsulated between thin layers of $\mathrm{hBN}$, as presented in Fig. $4 \mathrm{a}, \mathrm{b}$, respectively. In agreement with recent literature ${ }^{45-47}$, the successful encapsulation leads to an overall narrowing of the exciton resonances. Most importantly, however, the changes in the $n=2$ linewidth by a factor of 20 , from almost 

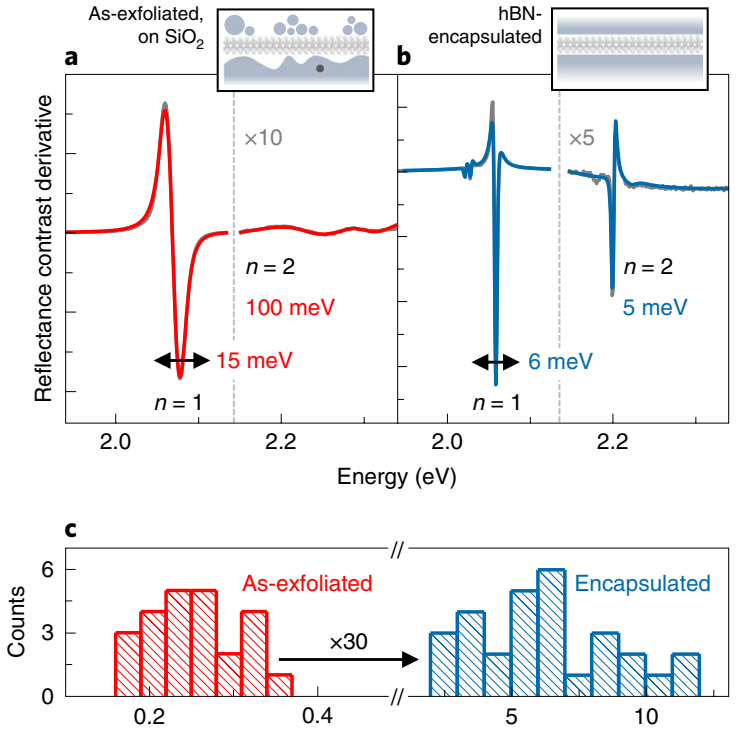

Exciton room-temperature diffusion coefficient $\left(\mathrm{cm}^{2} \mathrm{~s}^{-1}\right)$

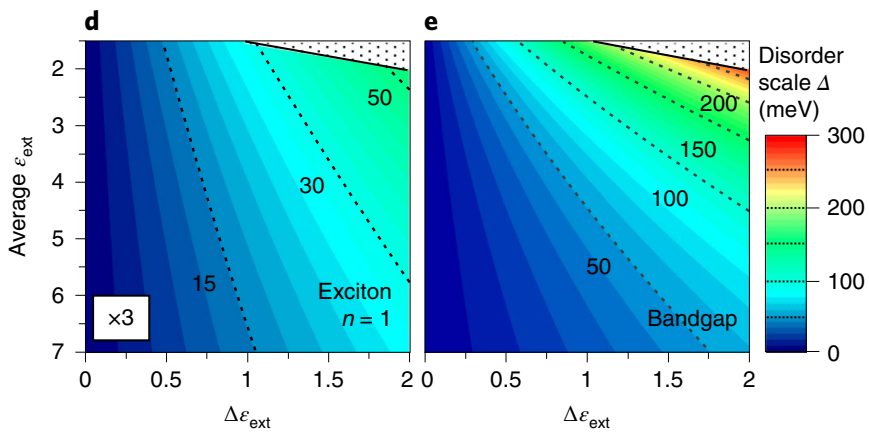

Fig. 4 | Impact of dielectric disorder on optics and exciton transport in heterostructures. $\mathbf{a}, \mathbf{b}$, Low-temperature reflectance contrast derivatives of bare $\mathrm{WS}_{2}$ on a $\mathrm{SiO}_{2}$ substrate (a) and encapsulated between thin layers of hBN (b). The measured data and corresponding simulated multi-peak spectra are shown by grey and coloured lines, respectively. c, Histogram of exciton diffusion coefficients at room temperature obtained from as-exfoliated and hBN-encapsulated samples. $\mathbf{d}, \mathbf{e}$, Calculated disorder scales (defined as maximum-to-minimum energies) for the ground-state exciton (d) and bandgap (e) as a function of the fluctuations of the external screening $\Delta \varepsilon_{\text {ext }}$ and average value $\varepsilon_{\text {ext }}$. The colour scale of the energies in figure $\mathbf{d}$ is rescaled by a factor of 3 for better contrast.

$100 \mathrm{meV}$ to $5 \mathrm{meV}$, are much larger than the decrease of the $n=1$ broadening (on the order of $10 \mathrm{meV}$ ) by a factor of about 2 to 3 (see Supplementary Section 6 for the $\mathrm{WSe}_{2}$ results). These observations strongly support the resonance narrowing originating from essentially removing the dielectric disorder, strongly decreasing the magnitude of free-particle bandgap fluctuations, and thus leading to extreme narrowing of the higher excited exciton states in particular. We further demonstrate that one can also obtain large-area $\left(>200 \mu \mathrm{m}^{2}\right)$ encapsulated heterostructures with only small variations of the dielectric screening across several tens of micrometres (Supplementary Sections 8 and 9). Finally, we note that, in the discussed context, electronic hybridization effects between $\mathrm{WS}_{2}$ and hBN should be negligible, as recently shown in angle-resolved photoemission experiments ${ }^{48}$.

To illustrate the implications of the studied phenomena for exciton transport at room temperature and under ambient conditions, we performed time- and spatially resolved photoluminescence measurements on a series of as-exfoliated and hBN-encapsulated
$\mathrm{WS}_{2}$ samples (see Supplementary Section 10 and ref. ${ }^{49}$ for details). The latter were preselected for narrow exciton linewidths of both ground and excited states observed across relatively large areas of many $\mu \mathrm{m}^{2}$ to ensure successful encapsulation and good interlayer contact. Effective exciton diffusion coefficients were directly obtained from the time dependence of the emission profiles in the linear regime with pump energy densities below $0.1 \mu \mathrm{J} \mathrm{cm}^{-2}$. The main results are presented as a histogram of measured diffusion coefficients in Fig. 4c. We find that the observed linewidth narrowing and mitigation of disorder in $\mathrm{hBN}$-encapsulated samples leads to a reproducible, consistent increase of the exciton propagation efficiency by a factor of about 30 . The absolute values of the diffusion coefficient in the range of $5-10 \mathrm{~cm}^{2} \mathrm{~s}^{-1}$ correspond to effective exciton mobilities on the order of $200-400 \mathrm{~cm}^{2} \mathrm{~V}^{-1} \mathrm{~s}^{-1}$, in close agreement with the conclusions from recent observations in hBN-encapsulated WSe ${ }_{2}\left(\right.$ ref. $\left.^{50}\right)$.

\section{Conclusions}

Fluctuations of the external dielectric screening $\varepsilon_{\text {ext }}$ along the inplane coordinates are found to provide characteristic energy scales for dielectric disorder $\Delta_{\mathrm{X}}^{\mathrm{b}}$ and $\Delta_{\text {gap }}$ for the exciton states and free carrier bandgap, respectively. Based on the observation of smooth, inhomogeneously broadened spectra, an ensemble of much smaller regions of variable screening is often probed in optical experiments with micrometre spot sizes. The lower limit for the spatial extent of dielectric disorder is then given by the exciton radii of several nanometres $^{19}$. Interestingly, even moderate fluctuations of the external screening already result in sizable energy scales of the dielectric disorder. Calculated characteristic scales for the ground-state exciton peak energy and bandgap size are presented in Fig. $4 \mathrm{~d}$,e as a function of the average value of $\varepsilon_{\text {ext }}$ and its fluctuations by $\pm \Delta \varepsilon_{\text {ext }} / 2$. The resulting random variations of the bandgap reach up to $300 \mathrm{meV}$ in a reasonable range of the dielectric screening parameters. Those of the ground-state exciton transition can be as high as $50 \mathrm{meV}$. These energy scales are sufficiently large to impact both optics and transport for a broad range of experimental conditions, including elevated temperatures and high carrier densities.

In conclusion, we have introduced the concept of dielectric disorder in semiconducting nanostructures using 2D materials as prototypical systems. We have presented the consequences of random fluctuations of the dielectric environment by monitoring the statistics and correlations of the exciton state energies and linewidths from experiments that are in close quantitative agreement with theoretical modelling. The importance of controlling the local dielectric homogeneity is further emphasized in the context of van der Waals heterostructures, including the capability of the hBN encapsulation to essentially remove dielectric disorder, facilitate pronounced linewidth narrowing of ground and excited states, and enable efficient transport. Overall, the demonstration of an alternative source of imperfections based entirely on the consequences of Coulomb interaction should have profound, direct implications for nanotechnology and fundamental materials science.

\section{Online content}

Any methods, additional references, Nature Research reporting summaries, source data, statements of code and data availability and associated accession codes are available at https://doi.org/10.1038/ s41565-019-0520-0.

Received: 28 November 2018; Accepted: 2 July 2019; Published online: 19 August 2019

\section{References}

1. Klingshirn, C. Semiconductor Optics, 3rd edn (Springer, 2007).

2. Fahey, P. M., Griffin, P. B. \& Plummer, J. D. Point defects and dopant diffusion in silicon. Rev. Mod. Phys. 61, 289-384 (1989). 
3. Singh, J. \& Bajaj, K. K. Role of interface roughness and alloy disorder in photoluminescence in quantum-well structures. J. Appl. Phys. 57, 5433-5437 (1985).

4. Nakamura, S. The roles of structural imperfections in InGaN-based blue light-emitting diodes and laser diodes. Science 281, 956-961 (1998).

5. Alivisatos, A. P., Harris, A. L., Levinos, N. J., Steigerwald, M. L. \& Brus, L. E. Electronic states of semiconductor clusters: homogeneous and inhomogeneous broadening of the optical spectrum. J. Chem. Phys. 89, 4001 (1988).

6. Tersoff, J. \& Legoues, F. K. Competing relaxation mechanisms in strained layers. Phys. Rev. Lett. 72, 3570-3573 (1994).

7. Haug, H. \& Koch, S. W. Quantum Theory of the Optical and Electronic Properties of Semiconductors, 5th edn (World Scientific, 2009).

8. Rytova, N. S. Screened potential of a point charge in a thin film. Proc. MSU Phys. Astron. 3, 30 (1967).

9. Keldysh, L. V. Coulomb interaction in thin semiconductor and semimetal films. JETP Lett. 29, 658-661 (1979).

10. Stier, A. V., Wilson, N. P., Clark, G., Xu, X. \& Crooker, S. A. Probing the influence of dielectric environment on excitons in monolayer $\mathrm{WSe}_{2}$ : insight from high magnetic fields. Nano Lett. 16, 7054-7060 (2016).

11. Rösner, M. et al. Two-dimensional heterojunctions from nonlocal manipulations of the interactions. Nano Lett. 16, 2322-2327 (2016).

12. Raja, A. et al. Coulomb engineering of the bandgap and excitons in two-dimensional materials. Nat. Commun. 8, 15251 (2017).

13. Cho, Y. \& Berkelbach, T. C. Environmentally sensitive theory of electronic and optical transitions in atomically thin semiconductors. Phys. Rev. B 97, 041409 (2018).

14. Winther, K. T. \& Thygesen, K. S. Band structure engineering in van der Waals heterostructures via dielectric screening: the GW method. 2D Mater. 4, 025059 (2017).

15. Utama, M. I. B. et al. A dielectric-defined lateral heterojunction in a monolayer semiconductor. Nat. Electron. 2, 60-65 (2019).

16. Haigh, S. J. et al. Cross-sectional imaging of individual layers and buried interfaces of graphene-based heterostructures and superlattices. Nat. Mater. 11, 764-767 (2012).

17. Zhou, C., Yang, W. \& Zhu, H. Mechanism of charge transfer and its impacts on Fermi-level pinning for gas molecules adsorbed on monolayer $\mathrm{WS}_{2}$. J. Chem. Phys. 142, 214704 (2015).

18. Berkelbach, T. C. \& Reichman, D. R. Optical and excitonic properties of atomically thin transition-metal dichalcogenides. Annu. Rev. Condens. Matter Phys. 9, 379-396 (2018).

19. Wang, G. et al. Colloquium: excitons in atomically thin transition metal dichalcogenides. Rev. Mod. Phys. 90, 021001 (2018)

20. Li, Y. \& Heinz, T. F. Two-dimensional models for the optical response of thin films. 2D Mater. 5, 025021 (2018).

21. Berghäuser, G. \& Malic, E. Analytical approach to excitonic properties of $\mathrm{MoS}_{2}$. Phys. Rev. B 89, 125309 (2014).

22. Berkelbach, T. C., Hybertsen, M. S. \& Reichman, D. R. Bright and dark singlet excitons via linear and two-photon spectroscopy in monolayer transition-metal dichalcogenides. Phys. Rev. B 92, 085413 (2015).

23. Hill, H. M. et al. Observation of excitonic Rydberg states in monolayer $\mathrm{MoS}_{2}$ and $\mathrm{WS}_{2}$ by photoluminescence excitation spectroscopy. Nano Lett. 15, 2992 (2015).

24. Brem, S. et al. Intrinsic lifetime of higher excitonic states in tungsten diselenide monolayers. Nanoscale 11, 12381-12387 (2019).

25. Selig, M. et al. Excitonic linewidth and coherence lifetime in monolayer transition metal dichalcogenides. Nat. Commun. 7, 13279 (2016).

26. Kazimierczuk, T., Fröhlich, D., Scheel, S., Stolz, H. \& Bayer, M. Giant Rydberg excitons in the copper oxide $\mathrm{Cu}_{2} \mathrm{O}$. Nature 514, 343-347 (2014).

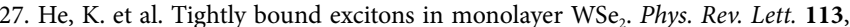
026803 (2014)

28. Chernikov, A. et al. Exciton binding energy and nonhydrogenic Rydberg series in monolayer WS. Phys. Rev. Lett. 113, 076802 (2014).

29. Conley, H. J. et al. Bandgap engineering of strained monolayer and bilayer $\mathrm{MoS}_{2}$. Nano Lett. 13, 3626-3630 (2013).

30. Wang, Y. et al. Strain-induced direct-indirect bandgap transition and phonon modulation in monolayer $\mathrm{WS}_{2}$. Nano Res. 8, 2562-2572 (2015).

31. Roldán, R., Castellanos-Gomez, A., Cappelluti, E. \& Guinea, F. Strain engineering in semiconducting two-dimensional crystals. J. Phys. Condens. Matter 27, 313201 (2015).

32. Lloyd, D. et al. Band gap engineering with ultralarge biaxial strains in suspended monolayer $\mathrm{MoS}_{2}$. Nano Lett. 16, 5836-5841 (2016).

33. Shin, B. G. et al. Indirect bandgap puddles in monolayer $\mathrm{MoS}_{2}$ by substrateinduced local strain. Adv. Mater. 28, 9378-9384 (2016).

34. Aslan, O. B., Deng, M. \& Heinz, T. F. Strain tuning of excitons in monolayer $\mathrm{WSe}_{2}$. Phys. Rev. B 98, 115308 (2018).
35. Niehues, I. et al. Strain control of exciton-phonon coupling in atomically thin semiconductors. Nano Lett. 18, 1751-1757 (2018).

36. Aslan, O. B. et al. Probing the optical properties and strain-tuning of ultrathin $\mathrm{Mo}_{1-x} \mathrm{~W}_{x} \mathrm{Te}_{2}$. Nano Lett. 18, 2485-2491 (2018).

37. Mak, K. F. et al. Tightly bound trions in monolayer $\mathrm{MoS}_{2}$. Nat. Mater. 12, 207-211 (2013)

38. Ross, J. S. et al. Electrical control of neutral and charged excitons in a monolayer semiconductor. Nat. Commun. 4, 1474 (2013).

39. Chernikov, A. et al. Electrical tuning of exciton binding energies in monolayer $\mathrm{WS}_{2}$. Phys. Rev. Lett. 115, 126802 (2015).

40. Borsenberger, P. M. \& Bässler, H. The role of polar additives on charge transport in molecularly doped polymers. Phys. Status Solidi B 170, 291-302 (1992).

41. Cui, X. et al. Multi-terminal transport measurements of $\mathrm{MoS}_{2}$ using a van der Waals heterostructure device platform. Nat. Nanotechnol. 10, 534-540 (2015).

42. Dean, C. R. et al. Boron nitride substrates for high-quality graphene electronics. Nat. Nanotechnol. 5, 722-726 (2010).

43. Cadiz, F. et al. Excitonic linewidth approaching the homogeneous limit in $\mathrm{MoS}_{2}$-based van der Waals heterostructures. Phys. Rev. X 7, 021026 (2017).

44. Ajayi, O. A. et al. Approaching the intrinsic photoluminescence linewidth in transition metal dichalcogenide monolayers. 2D Mater. 4, 031011 (2017).

45. Courtade, E. et al. Charged excitons in monolayer $\mathrm{WSe}_{2}$ : experiment and theory. Phys. Rev. B 96, 085302 (2017).

46. Manca, M. et al. Enabling valley selective exciton scattering in monolayer $\mathrm{WSe}_{2}$ through upconversion. Nat. Commun. 8, 14927 (2017).

47. Stier, A. V. et al. Magnetooptics of exciton Rydberg states in a monolayer semiconductor. Phys. Rev. Lett. 120, 057405 (2018).

48. Katoch, J. et al. Giant spin-splitting and gap renormalization driven by trions in single-layer $\mathrm{WS}_{2} / \mathrm{h}-\mathrm{BN}$ heterostructures. Nat. Phys. 14, 355-359 (2018).

49. Kulig, M. et al. Exciton diffusion and halo effects in monolayer semiconductors. Phys. Rev. Lett. 120, 207401 (2018).

50. Cadiz, F. et al. Exciton diffusion in $\mathrm{WSe}_{2}$ monolayers embedded in a van der Waals heterostructure. Appl. Phys. Lett. 112, 152106 (2018)

\section{Acknowledgements}

The authors thank M.M. Glazov and D.R. Reichman for interesting and helpful discussions. The authors thank all our colleagues who have contributed to individual sample preparation: G. Arefe, H.M. Hill, J. Hone, P. Nagler, T. Korn, A.F. Rigosi, J. Yu, A. van der Zande and X. Zhang. Financial support by the DFG via Emmy Noether grant no. CH 1672/1-1 and Collaborative Research Center SFB 1277 (B05) is acknowledged. This research was supported by the AMOS programme at the SLAC National Accelerator Laboratory within the Chemical Sciences Geosciences and Biosciences Division for data analysis, by the National Science Foundation under grant no. DMR-1708457 for the fabrication of samples on h-BN, and by the Gordon and Betty Moore Foundation's EPiQS programme through grant no. GBMF4545 for spectroscopic measurements. A.R. acknowledges funding through the Heising-Simons Junior Fellowship within the Kavli Energy NanoScience Institute at the University of California, Berkeley. L.W. acknowledges support by the Alexander von Humboldt Foundation. This work was partially supported by the US-Israel Binational Science Foundation grant no. BSF2016362 (Y.C. and T.C.B.). The Flatiron Institute is a division of the Simons Foundation. The project has also received funding from the Swedish Research Council (VR) and the European Union's Horizon 2020 research and innovation programme under grant no. 785219 (Graphene Flagship). Growth of hBN crystals was supported by the Elemental Strategy Initiative conducted by the MEXT, Japan and the CREST (JPMJCR15F3), JST

\section{Author contributions}

A.C. and A.R. conceived the idea, with L.W., T.C.B and T.F.H. providing additional input A.R., L.W., J.Z., J.D.Z., M.K. and A.C. prepared the samples, carried out the experiments and analysed the data. Y.C. and T.C.B. performed theoretical calculations of the dielectric effects. S.B. and E.M. performed calculations of the exciton-phonon scattering rates. T.T and K.W. provided high-quality hBN crystals. A.C., A.R. and L.W. wrote the manuscript with input from all authors.

\section{Competing interests}

The authors declare no competing interests.

\section{Additional information}

Supplementary information is available for this paper at https://doi.org/10.1038/ s41565-019-0520-0.

Reprints and permissions information is available at www.nature.com/reprints. Correspondence and requests for materials should be addressed to A.R. or A.C. Publisher's note: Springer Nature remains neutral with regard to jurisdictional claims in published maps and institutional affiliations.

(C) The Author(s), under exclusive licence to Springer Nature Limited 2019 


\section{Methods}

Sample preparation. The monolayer $\mathrm{WS}_{2}$ and $\mathrm{WSe}_{2}$ samples used to obtain the statistics of the exciton linewidths at cryogenic temperatures were directly exfoliated from commercially available bulk crystals (obtained from 2Dsemiconductors and HQgraphene) either onto $\mathrm{SiO}_{2} / \mathrm{Si}$ or fused-silica substrates No additional processing was applied and the samples were studied in their pristine as-exfoliated state. The monolayer $\mathrm{WS}_{2}$ samples for the comparative study on PDMS and hBN substrates were also produced from commercially available bulk $\mathrm{WS}_{2}$ crystals (obtained from HQgraphene). First, crystals on scotch tape were directly exfoliated onto PDMS. Subsequently, samples on hBN were fabricated by direct exfoliation of separate $\mathrm{hBN}$ and $\mathrm{WS}_{2}$ flakes onto PDMS that were then stamped onto graphene-covered fused-silica substrates. The fabrication was followed by an annealing step at $120-150^{\circ} \mathrm{C}$ for $1-2 \mathrm{~h}$ in vacuum. The data presented in Fig. $3 \mathrm{~b}$ in the main text were collected from 38 individual positions on seven different $\mathrm{WS}_{2}$ samples on $\mathrm{hBN}$ and from 21 individual positions on nine samples on PDMS. To obtain hBN-encapsulated samples, thin hBN flakes were first exfoliated on PDMS from bulk crystals (provided by T. Taniguchi and $\mathrm{K}$. Watanabe, NIMS), stamped on a $100^{\circ} \mathrm{C}$ preheated $\mathrm{SiO}_{2} / \mathrm{Si}$ substrate at ambient conditions, and subsequently annealed in high vacuum at $150^{\circ} \mathrm{C}$ for $4-5 \mathrm{~h}$. Monolayer WS $\mathrm{W}_{2}$ or WSe $\mathrm{W}_{2}$ flakes, exfoliated on PDMS, were then stamped on top of the hBN layers at $70^{\circ} \mathrm{C}$ substrate temperature and under ambient conditions. Samples with $\mathrm{WSe}_{2}$ were subsequently annealed again in high vacuum at $150^{\circ} \mathrm{C}$ for $4-5 \mathrm{~h}$, followed by the stamping of a thin $\mathrm{hBN}$ layer on top at $70^{\circ} \mathrm{C}$ substrate temperature. The resulting hBN-WSe $e_{2}-\mathrm{hBN}$ heterostructure was then annealed again in high vacuum at $150^{\circ} \mathrm{C}$ for $4-5 \mathrm{~h}$. In the case of $\mathrm{WS}_{2}$, the structure was covered by a thin $\mathrm{hBN}$ layer without any additional annealing.

Optical spectroscopy. Optical reflectance spectroscopy was performed on several set-ups using similar equipment and measurement procedures. In these experiments, the light from a spectrally broadband tungsten-halogen lamp was focused by $\mathrm{a} \times 40, \mathrm{NA}=0.6$ objective onto the sample. The resulting spot sizes were typically in the range of one to a few micrometres and the integrated incident power on the sample was on the order of several hundreds of $\mathrm{nW}$ or below. The reflected light was spectrally dispersed in a grating spectrometer and subsequently detected by a cooled charge-coupled device (CCD) camera. The count accumulation time of an individual frame was usually chosen to be close to the saturation threshold of the CCD chip, followed by an integration over tens to hundreds of frames to optimize the signal-to-noise ratio. The data in Fig. 3 were taken at room temperature and under ambient pressure. Spectra collected at cryogenic temperature were measured on samples mounted in an optical microscopy cryostat cooled either by liquid nitrogen or liquid helium.

Experimental data analysis. The measured reflectance signals were analysed in terms of reflectance contrast, defined as relative additional change of the sample reflectance with respect to the substrate reference. The peak linewidths were extracted from the reflection contrast spectra by appropriate fitting procedures. First, a model dielectric function was constructed as a sum of non-radiatively broadened Lorentz oscillators, each representing one of the main absorption features (see Supplementary Information for details). For the analysis of roomtemperature data in Fig. 3, Faddeeva functions were used to deconvolute homogeneous and inhomogeneous broadening contributions. The reflectivity of the stack was then calculated using this parameterized dielectric function and refractive indices of all other films from the literature, taking into account multilayer interference effects via the transfer matrix formalism. Finally, a comparison between the measured and calculated reflection spectra was used to optimize the parameters of the oscillators in the dielectric function, including oscillator strengths, central peak energies and linewidths.

Calculations of exciton and bandgap energies. To describe the effect of the environmental dielectric screening on the bandgap and excitons, the monolayer was treated as an infinite slab of finite thickness surrounded by a continuum dielectric. The screened Coulomb interaction was derived using the method of image charges, as elaborated in Supplementary Section 7. The change in the bandgap of the monolayer compared to its value in the bulk was evaluated as the sum of the self-energy of a hole for the valence band and an electron for the conduction band. The Schrödinger equation with an effective mass Hamiltonian was solved to calculate the exciton states and their binding energies. The eigenfunctions and eigenvalues of the exciton Hamiltonian were found via diagonalization in a basis of radial real-space grid points. The results of the above calculations depend on the environmentally screened interaction, modifying the quasiparticle bandgap, the exciton binding energy and the optical gap defined as the peak energy of the exciton ground-state resonance.

Calculations of exciton-phonon scattering. To assess the contributions from homogeneous broadening of the ground and excited exciton states on the level of microscopic theory, semiconductor Bloch equations were numerically solved in the low density limit based on material parameters from ab initio calculations. The Coulomb interaction was treated on a Hartree-Fock level where the interaction strength was determined by the Rytova-Keldysh potential for $2 \mathrm{D}$ films. The dielectric susceptibility of the monolayer was expressed in the excitonic basis, where the excitonic wavefunctions and the eigen energies were determined by the solution of the Wannier equation. Homogeneous linewidths of the exciton transitions were microscopically calculated by taking into account radiative and phonon-induced dephasing, obtained within a second-order Born-Markov approximation for exciton-phonon correlations. Both intra- and intervalley scattering processes with acoustic and optical phonons were included for the whole excitonic Rydberg series for intra- as well as intervalley excitons. From the microscopically calculated susceptibility, the reflectance contrast of the monolayer on the fused-silica substrate was obtained. Additional details are presented in Supplementary Section 6.

\section{Data availability}

All relevant data are available from the authors upon reasonable request. 